Following adjustment for the sodium excretion index, an indirect measure of salt intake, there association with meat was no longer significant.

Conclusion Inadequate control of BP in patients who were being treated with anti-hypertensive drugs, even in a community assisted by a primary care program, was independently associated with modifiable factors including salt consumption and BMI. An association with skin colour and serum creatinine was also observed.

\section{SP1-71 HOW DOES THE AGE INFLUENCE ON THE RELATIONSHIP BETWEEN CENTRAL BLOOD PRESSURE AND CARDIOVASCULAR RISK FACTORS? CROSS-SECTIONAL ANALYSIS OF NAGAHAMA ZERO-JI PREVENTIVE COHORT PROJECT IN JAPAN}

doi:10.1136/jech.2011.142976n.48

${ }^{1} \mathrm{~S}$ Tokumoto, ${ }^{*}{ }^{1} \mathrm{Y}$ Takahashi, ${ }^{1} \mathrm{~T}$ Ishizaki, ${ }^{2} \mathrm{~K}$ MIYAKI, ${ }^{1} \mathrm{~T}$ Nakayama. ${ }^{1}$ Department of Health Informatics, Kyoto University School of Public Health, Kyoto City, Japan; ${ }^{2}$ National Center for Global Health and Medicine, Tokyo, Japan

Introduction Blood pressure is a well established cardiovascular risk factor. In addition to traditional blood pressure indexes such as SBP and DBP, estimation of central SBP (cSBP) has become available recently. The characteristics of cSBP have not been fully described. This study investigated the relationship between cSBP and known cardiovascular risk factors according to age group and comparing this with four traditional blood pressure indexes, SBP, DBP, PP and MBP.

Methods This study examined baseline cross-sectional data from 4375 healthy men and women aged $30-74$ years old who participated in a community-based cohort study conducted from 2008 to 2009. Linear regression analyses were carried out on the five blood pressure indexes adjusting for sex, age, BMI, triglycerides, HDLcholesterol, LDL-cholesterol, $\mathrm{HbA1c}$, state of anti-hypertensive medication, smoking, alcohol Intake, exercise, past history of cardiovascular diseases, ABI and cardio-ankle vascular index. We analysed the following two models: including these risk factors without sex and age (model 1); including age groups (age $<50$ / age $\geq 50$ ) (model 2). Subgroup analyses according to age group and sex were also performed.

Results In model 1 the $\mathrm{R}^{2}$ for sBP was 0.356 , and in model $2,0.385$. Incremental $\mathrm{R}^{2}$ was 0.028 between the models. Subgroup analyses showed five blood pressure indexes were explained by the total variances of known cardiovascular risk factors, more so for the younger than the older age group which was more apparent for cSBP than for the other blood pressure indexes.

Conclusion Known cardiovascular risk factors are associated with cSBP. This association is greater in younger $($ age $<50)$ than in older (age $\geq 50$ ) patients.

\section{SP1-72 FACTORS ASSOCIATED WITH DEPRESSION AMONG ELDERLY IN KARACHI PAKISTAN: A MATCHED CASE CONTROL STUDY}

doi:10.1136/jech.2011.142976n.49

${ }^{1} \mathrm{~K}$ Nanji, ${ }^{2} \mathrm{M}$ Kadir, ${ }^{3} \mathrm{~K}$ Ahmed, ${ }^{4} \mathrm{H}$ Naqvi. ${ }^{1}$ Department of Family Medicine, The Aga Khan University, Karachi, Pakistan; ${ }^{2}$ Department of Community Health Sciences, The Aga Khan University, Karachi, Pakistan; ${ }^{3}$ Department of Opthalmology, The Aga Khan University, Karachi, Pakistan; ${ }^{4}$ Department of Psychiatry, The Aga Khan University, Karachi, Pakistan

Introduction The increase in life expectancy due to epidemiologic transition has brought about increased numbers of certain mental illnesses, namely depression. There is scarcity of analytical base epidemiological studies on this issue. This study was conducted to identify factors associated with depression among elderly $(>60)$ in Karachi, Pakistan.

Method A multicentre matched case control study was conducted. A total of 234 depressed cases and 468 non-depressed controls (1case:2controls) were selected from four tertiary care hospitals in Karachi. Cases were recruited from psychiatric OPD whereas; controls were recruited from other OPD's (except psychiatric) and were individually matched on age and gender with cases. Ascertainment of cases and controls was done by psychiatrist and Geriatric Depression Scale. There was substantial agreement between the psychiatrists for diagnosing depression ( $\kappa$ : 0.66). A pilot tested structured questionnaire was administered and analysis was performed through conditional logistic regression using SPSS 11.5

Results Risk factors for depression were, living in nuclear family system, (mOR: 4, CI 2.1 to 4.9), self reported difficult situation during past 1 year (mOR: 9, CI 6.0 to 19.7), hearing and/or visual impairment (mOR: 5, CI 2.0 to 9.8), presence of $>2$ NCD (mOR: 2 , CI 1.0 to 3.4). However, interacting with friends or relatives had a protective effect against depression (mOR adj: $0.3,95 \%$ CI 0.3 to 0.7$)$.

Conclusion Interventions should be taken to reduce modifiable factors and non-modifiable factors should be used to identify high risk group for primary prevention. However factors identified through this study may vary in its association with depression across different communities.

\section{SP1-73 THE PREVALENCE OF COGNITIVE IMPAIRMENT AND ITS ASSOCIATION WITH SOCIAL SUPPORT IN ELDERLY PEOPLE IN KARACHI, PAKISTAN}

doi:10.1136/jech.2011.142976n.50

${ }^{1} \mathrm{M}$ Bhamani, ${ }^{1} \mathrm{~K}$ Nanji.* ${ }^{1}$ Department of Community Health Sciences-The Aga Khan University, Karachi, Pakistan; ${ }^{2}$ Department of Family Medicine-The Aga Khan University, Karachi, Pakistan

Introduction Cognitive impairment is one of the commonest mental health problems in the elderly. The objective of this study was to estimate the prevalence of cognitive impairment in the elderly (age $>60$ years) population in Karachi, Pakistan and to determine the association between social support and cognitive impairment in this group.

Methods A population based cross sectional study of the elderly in Karachi Pakistan was carried out between November 2010 and March 2011. Participants were randomly recruited in 30 clusters from 18 towns in Karachi using a multi-stage cluster sampling technique. Face-to-Face interviews were conducted with 730 eligible participants using structured questionnaires. The prevalence of cognitive impairment was assessed through Mini Mental State Examination (MMSE). For illiterate participants cognitive impairment was defined as a score of $<21$; for literate $<23$. Data analysis is underway using SPSS 16. Logistic regression analysis will be used to determine the association between social support and cognitive impairment.

Results This study is currently in progress. We anticipate that prevalence of cognitive impairment will be higher and will be attributable to the social support received by the elderly.

Conclusion Conclusion will be based on the results of this study. However, we suggest that the younger generation should be sensitised towards their responsibilities for the elderly and the government should also formulate effective health policies for this group. Further, longitudinal studies should be planned to identify other factors that are associated with cognitive impairment in the elderly. 